\title{
Three Children Treated with Direct-acting Antivirals for Chronic Hepatitis C Virus Genotype 1b Infection
}

\author{
Kazuki Ohya, Tomokazu Kawaoka, Michio Imamura, Kei Morio, Takashi Nakahara, \\ Eisuke Murakami, Masami Yamauchi, Akira Hiramatsu, Masataka Tsuge, \\ Hiroshi Aikata and Kazuaki Chayama
}

\begin{abstract}
:
Although direct-acting antivirals (DAAs) have significantly increased the sustained virological response (SVR) rates in chronic hepatitis $\mathrm{C}$ virus (HCV)-infected adult patients, the efficacy and safety for children remain unclear. We herein report three HCV-infected children who received DAA treatment. The patients were girls 10-13 years old who had been infected with genotype $1 \mathrm{~b}$ HCV by vertical transmission based on a phylogenetic tree analysis. Two patients were treated with 12 weeks of ombitasvir/paritaprevir/ritonavir, and the other patient was treated with 8 weeks of glecaprevir/pibrentasvir. All children received DAA doses that were similar to the dosages for adult patients. None developed adverse events, and all children achieved an SVR.
\end{abstract}

Key words: HCV, DAA, child

(Intern Med 59: 941-944, 2020)

(DOI: 10.2169/internalmedicine.3824-19)

\section{Introduction}

The current standard of care for chronic hepatitis $\mathrm{C}$ virus $(\mathrm{HCV})$ infections in adults includes several all-oral regimens comprising direct-acting antivirals (DAAs) that specifically target HCV. DAAs are highly effective and have a favorable safety profile $(1,2)$. Therefore, almost all adult patients have been able to achieve HCV eradication. However, children with chronic $\mathrm{HCV}$ infection have fewer treatment options than adults. Until recently, the standard of care for chronic $\mathrm{HCV}$ pediatric infections was interferon or peginterferon and ribavirin therapy for 24 or 48 weeks, a regimen that requires subcutaneous injections and is associated with major side effects, including growth impairment (3-7).

The expansion of HCV treatment options to include alloral DAA regimens for children and adolescents should improve the accessibility of treatment worldwide. We treated three 10- to 13-year-old HCV-infected children using DAA regimens, and a sustained virological response (SVR) was achieved in all patients.

We herein report the efficacy and safety of DAA treat- ments for HCV-infected children. Written informed consent was obtained from all of the patients' parents. This study was approved by the Institutional Review Board of Hiroshima University and conducted in accordance with the Declaration of Helsinki.

\section{Case Reports}

The baseline characteristics of the three patients are shown in Table. All patients were female children 10-13 years of age. Cases 1 and 2 had been followed from birth by clinicians in the Department of Pediatrics, who consulted us about antiviral therapy. Case 3 underwent a health checkup at our hospital prior to dental treatment and was found to be positive for $\mathrm{HCV}$ antibody. All children were infected with HCV genotype $1 \mathrm{~b}$, and all of their mothers were also infected with $\mathrm{HCV}$ genotype $1 \mathrm{~b}$. A phylogenetic tree analysis showed similar HCV strains between the child and mother in all cases, suggesting that HCV infection had resulted from vertical transmission in these cases (Figure).

Based on the results of ultrasonography, transient elastography, and laboratory investigations, all patients were deter- 
Table. Clinical Characteristics of Three Children with Chronic Hepatitis C Virus (HCV) Genotype 1b Infection.

\begin{tabular}{|c|c|c|c|}
\hline & Case 1 & Case 2 & Case 3 \\
\hline Age & $10 \mathrm{y} 7 \mathrm{~m}$ & $13 \mathrm{y} 11 \mathrm{~m}$ & $10 \mathrm{y} 8 \mathrm{~m}$ \\
\hline Sex & Female & Female & Female \\
\hline Body height (m) & 1.42 & 1.51 & 1.37 \\
\hline Body weight (kg) & 48.9 & 38.0 & 30.9 \\
\hline History of anti-HCV therapy & No & No & No \\
\hline Leukocyte count $\left(/ \mathrm{mm}^{3}\right)$ & 7,480 & 5,260 & 8,720 \\
\hline Hemoglobin $(g / d L)$ & 13.9 & 13.1 & 13.8 \\
\hline Platelet count $\left(\times 10^{4} / \mu \mathrm{L}\right)$ & 24.6 & 25.4 & 35.9 \\
\hline Prothrombin activity (\%) & 98 & 97 & 97 \\
\hline Total bilirubin (mg/dL) & 0.7 & 1.1 & 0.5 \\
\hline Aspartate aminotransferase (U/L) & 38 & 18 & 33 \\
\hline Alanine aminotransferase (U/L) & 52 & 20 & 19 \\
\hline Albumin $(\mathrm{g} / \mathrm{dL})$ & 4.2 & 4.3 & 4.7 \\
\hline Alpha-fetoprotein $(\mathrm{ng} / \mathrm{mL})$ & 0.9 & 2.2 & 1.6 \\
\hline FIB4 index & 0.22 & 0.21 & 0.22 \\
\hline Fibroscan $(\mathrm{kPa})$ & 5.3 & 3.7 & 3.0 \\
\hline HCV genotype in children & $1 b$ & $1 b$ & $1 \mathrm{~b}$ \\
\hline $\mathrm{HCV}$ genotype in mothers & $1 b$ & $1 b$ & $1 b$ \\
\hline HCV RNA (log IU/mL) & 5.2 & 4.9 & 5.2 \\
\hline NS3-D168 & Wild & Wild & Wild \\
\hline NS5A-L31 & Wild & $\operatorname{Mix}(L>>I / V)$ & Wild \\
\hline NS5A-Y93 & Wild & Wild & Wild \\
\hline Regimens of DAAs & $\mathrm{OBV}+\mathrm{PTV} / \mathrm{r}$ & $\mathrm{OBV}+\mathrm{PTV} / \mathrm{r}$ & GLE+PIB \\
\hline \multirow[t]{2}{*}{ Doses of DAAs } & OBV $25 \mathrm{mg}$ & OBV $25 \mathrm{mg}$ & GLE $300 \mathrm{mg}$ \\
\hline & $\begin{array}{l}\text { PTV } 150 \mathrm{mg} \\
\text { r } 100 \mathrm{mg}\end{array}$ & $\begin{array}{l}\text { PTV } 150 \mathrm{mg} \\
\text { r } 100 \mathrm{mg}\end{array}$ & PIB $120 \mathrm{mg}$ \\
\hline
\end{tabular}

$\mathrm{OBV}+\mathrm{PTV} /$ r: ombitasvir+paritaprevir/ritonavir, GLE+PIB: glecaprevir+pibrentasvir

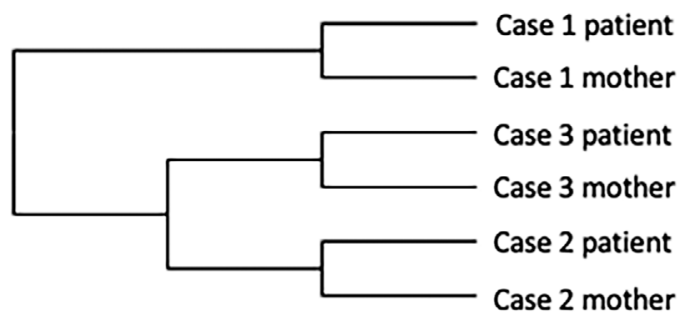

Tree name: GENETYX

OTU count: 6

Constructed by:

Construction date: 2019

Method = UPGMA

Distance $=$ Kimura's 2 parameter

Figure. A phylogenetic tree analysis of samples from three children and their mothers. A similar HCV strain infected each patient and mother pair.

mined to have chronic hepatitis, and cirrhosis was ruled out. In a laboratory investigation, we established the cut-off value of alanine aminotransferase (ALT) $>30$ (U/L) to determine whether or not patients had chronic hepatitis. Similarly, the findings of ultrasonography were also considered (the roughness of the liver parenchyma and dullness of the liver edge). Transient elastography and the FIB4 index were mainly used to determine whether the patients had chronic hepatitis or liver cirrhosis. The cut-off values of transient elastography and the FIB4 index were $\geq 10 \mathrm{kPa}$ and $\geq 3.25$, respectively. Case 1 had a high ALT level, and cases 2 and 3 had findings of chronic hepatitis on ultrasonography.

The amino acid sequences of the regions encompassing NS3-D168, NS5A-L31, and NS5A-Y93 were determined by the Invader assay for single nucleotide polymorphism genotyping. Resistance-associated variants (RAVs) were not detected in cases 1 and 3, while NS5A-L31I/V RAVs were detected in case 2 .

All children received DAA treatment with sufficient explanation provided to their parents. Cases 1 and 2 were treated with ombitasvir and paritaprevir plus ritonavir (OBV/ PTV/r) for 12 weeks, and case 3 was treated with glecaprevir plus pibrentasvir (GLE/PIB) for 8 weeks. Cases 1, 2, and 3 weighed 48.9, 38.0, and $30.9 \mathrm{~kg}$, respectively, and received the same dose of DAAs used for adult patients.

The serum HCV RNA levels decreased to below the detectable limit according to the COBAS TaqMan HCV test (Roche Diagnostics, Tokyo, Japan) at 4 weeks after the initiation of treatment in all patients. All patients completed treatment without a reduction in the DAA dose. Serum HCV RNA remained negative after the completion of treatment, and all patients showed an SVR at 12 and 24 weeks after the completion of treatment. None of the patients developed 
serious adverse events or laboratory abnormalities, such as elevated aminotransferase and bilirubin levels, during the treatment and follow-up periods. Pruritus (case 1), flu-like symptom (case 2), and viral enteritis (case 3) were reported; however, these conditions were considered by the investigator to be unrelated to study treatment.

\section{Discussion}

At present, an estimated 115-185 million individuals have serologic evidence of $\mathrm{HCV}$ infection, including roughly 11 million children under 15 years of age $(4,8)$. Pediatric HCV infection differs from adult $\mathrm{HCV}$ infection in several respects. Pediatric HCV infections occur mainly through vertical transmission during childbirth in developed countries, whereas it still occurs through horizontal transmission from adults in developing countries (9). Infections acquired through vertical transmission affect $3-10 \%$ of infants from HCV-infected mothers (10). Garazzino et al. reported on the natural history of vertically acquired $\mathrm{HCV}$ infection and showed that $27 \%$ of patients with vertically acquired HCV infection spontaneously achieved sustained viral clearance (SVC); the predictive factor associated with SVC is infection with HCV genotype 3 (11). Similarly, Mizuochi et al. also reported that the prevalence of SVC was $9 \%$ in 348 Japanese children who were enrolled their study (12).

In Japanese adults, HCV genotype $1 \mathrm{~b}$ infection is predominant, and genotype 3 infection is extremely rare (8). In fact, almost all patients infected with HCV genotype 1 or 2 in Mizuochi et al.'s report and all three children in the present study were infected with HCV genotype 1b (12). However, the prevalence of HCV genotype in Japan has recently changed; that of genotype 1 is decreasing, and that of genotype 2 is increasing, reaching $50 \%$ in individuals born after 1970 (13). In accordance with the change in the prevalence of HCV genotype in adult patients, the prevalence of genotype 2 is also increasing in Japanese children with HCV and is thought to be mainly acquired by vertical transmission (12).

Until recently, the treatment of pediatric patients with $\mathrm{HCV}$ infections has been controversial because the standard of care, pegylated interferon (PEG-IFN) and weight-based ribavirin, is associated with major side effects, including growth impairment and poor tolerability $(4,5,7)$. However, Suzuki et al. reported that $\mathrm{HCV}$-infected Japanese children tolerate PEG-IFN therapy well (14). DAA therapy has several advantages over PEG-IFN therapy, including a shorter duration of therapy ( 8 weeks vs. at least 24 weeks) and a noninvasive approach (oral therapy vs. frequent hospital visits and subcutaneous injections). Three active multicenter, all-oral, interferon-free clinical trials examining DAA use for children and adolescents have been initiated $(6,15)$, and DAA therapies such as sofosbuvir plus ledipasvir (SOF/ LDV) and SOF plus ribavirin have shown high effectiveness and safety for adolescents infected with $\mathrm{HCV}$ genotypes 1 and 2 or 3 , respectively $(16,17)$. Based on the results of these trials, guidance documents on the treatment of chronic $\mathrm{HCV}$ infection in children were recently established by the American Association for the Study of Liver Diseases (AASLD) and the Infectious Diseases Society of America (IDSA) (18). In 2017, updated Swedish consensus guidelines that are slightly different from the guidance documents were also published (19). The two guidance documents and the guidelines recommend combination treatment of SOF/ LDV for children older than 12 years of age who have $\mathrm{HCV}$ genotype 1 infection. Unfortunately, OBV/PTV/r was not available for use in the present cases because its marketing had been stopped. Because chronic HCV infection is associated with complications such as hepatic fibrosis, cirrhosis, and hepatocellular carcinoma (20), the introduction of these guidelines was quite helpful.

The existence of RAVs is strongly associated with the response to DAA treatment in $\mathrm{HCV}$-infected patients. In the present study, although pretreatment NS5A-L31I/V RAVs were detected in case 2, all patients achieved an SVR. Similarly, a previous clinical trial using $\mathrm{SOF} / \mathrm{LDV}$ resulted in a high SVR rate for genotype $1 \mathrm{HCV}$-infected adolescents, including patients with NS5A RAVs (16). Concerning RAV detection, Honda et al. reported that the semiquantitative polymerase chain reaction (PCR)-Invader assay is a highly sensitive method for determining the presence of pretreatment minor NS5A variants (21). Further studies on the relationship between DAA efficacy and RAVs in children and the optimal method for determining the presence of RAVs are needed.

AASLD and IDSA guidance documents recommend that children with HCV genotype 1 infection who are older than 12 years of age or weigh $\geq 35 \mathrm{~kg}$ and have chronic hepatitis or compensated cirrhosis be treated with SOF/LDV (18). To our knowledge, there have not been any reports on the treatment of children with OBV/PTV/r and GLE/PIB or on the treatment of children who are younger than 11 years of age. Regarding the dose setting for children, all patients were > $30 \mathrm{~kg}$ in this study. In the DORA study, which assessed the pharmacokinetics, the safety and efficacy of GLE/PIB in adolescents with chronic hepatitis $\mathrm{C}$ with a body weight between 32 and $109 \mathrm{~kg}$ also showed no clinically meaningful relationship between the body weight and serum GLE/PIB concentrations (22). Therefore, we used the adult dosage for the patients in our study.

All of the present patients achieved an SVR, and no serious adverse events were observed. At present, there are no definitive guidelines for the treatment of children 3-11 years of age with $\mathrm{HCV}$ infections or concerning the applicability of DAAs other than SOF/LDV for treatment. Although SOF/ LDV combination therapy showed high efficacy and safety for both adults and children, this treatment is difficult to use for patients with cardiac diseases. Our experience suggests that DAA treatment for $\mathrm{HCV}$-infected children under 12 years old is feasible and that DAAs other than SOF/LDV can be used.

The appropriate timing of antiviral therapy for HCV- 
infected children remains controversial. In the present study, although no patient had progressive liver fibrosis and no liver function impairment was noted on laboratory tests in cases 2 and 3, we decided to introduce antiviral therapy after obtaining sufficient informed consent from the parents. All patients and their parents wished to undergo treatment. In case 1 , the mother achieved an SVR early and stated her wish to have her daughter treated as well, while case 3's mother wished for her daughter to start treatment at the same time as her.

HCV eradication in children can afford a number of health benefits, such as a reduction in disease progression, resolution of emotional strain, and prevention of transmission. Furthermore, it was reported that $\mathrm{HCV}$ eradication by SOF/LDV therapy leads to improvements in the quality of life in adult patients (23). Based on these benefits, early antiviral therapy seems to be preferred for HCV-infected children, regardless of liver fibrosis and the liver function impairment. Further investigations will be necessary in order to determine the optimal antiviral therapy for children with chronic HCV infection, including the timing, appropriate drugs and doses, and duration of treatment.

In conclusion, treatment with recently developed DAAs for chronic $\mathrm{HCV}$ infection in adults has been dramatically effective. Clinical trials on DAAs for the treatment of children and adolescents with chronic HCV infection have also shown promising results. The availability of all-oral DAA regimens for children and adolescents with chronic $\mathrm{HCV}$ infection is expected to improve the care of patients who currently have limited treatment options.

\section{Author's disclosure of potential Conflicts of Interest (COI).}

Michio Imamura: Honoraria, Bristol-Myers Squibb; Research funding, Bristol-Myers Squibb. Masataka Tsuge: Research funding, Bristol-Myers Squibb. Kazuaki Chayama: Honoraria, MSD and Bristol-Myers Squibb; Research funding, AbbVie, Dainippon Sumitomo Pharma.

\section{References}

1. Chayama K, Suzuki F, Karino Y, et al. Efficacy and safety of glecaprevir/pibrentasvir in Japanese patients with chronic genotype 1 hepatitis $\mathrm{C}$ virus infection with and without cirrhosis. J Gastroenterol 53: 557-565, 2018.

2. Morio K, Imamura M, Kawakami Y, et al. Real-world efficacy and safety of daclatasvir and asunaprevir therapy for hepatitis $\mathrm{C}$ virusinfected cirrhosis patients. J Gastroenterol Hepatol 32: 645-650, 2017.

3. El-Guindi MA. Hepatitis C viral infection in children: updated review. Pediatr Gastroenterol Hepatol Nutr 19: 83-95, 2016.

4. Schwarz KB, Molleston JP, Jonas MM, et al. Durability of response in children treated with pegylated interferon alfa-2a \pm ribavirin for chronic hepatitis C. J Pediatr Gastroenterol Nutr 62: 93-96, 2016.

5. Jonas MM, Balistreri W, Gonzalez-Peralta RP, et al. Pegylated in- terferon for chronic hepatitis $\mathrm{C}$ in children affects growth and body composition: results from the pediatric study of hepatitis $\mathrm{C}$ (PEDS-C) trial. Hepatology 56: 523-531, 2012.

6. Yang CHT, Yoo ER, Ahmed A. The role of direct-acting antivirals in the treatment of children with chronic hepatitis C. J Clin Transl Hepatol 5: 59-66, 2017.

7. Tsunoda $\mathrm{T}$, Inui $\mathrm{A}$, Kawamoto $\mathrm{M}$, et al. Effects of pegylated interferon- $\alpha$-2a monotherapy on growth in Japanese children with chronic hepatitis C. Hepatol Res 44: 251-258, 2014.

8. Messina JP, Humphreys I, Flaxman A, et al. Global distribution and prevalence of hepatitis $\mathrm{C}$ virus genotypes. Hepatology 61: 7787, 2015

9. Pawlowska M, Domagalski K, Pniewska A, et al. What's new in hepatitis $\mathrm{C}$ virus infections in children? World J Gastroenterol 21: 10783-10789, 2015.

10. Yeung CY, Lee HC, Chan WT, et al. Vertical transmission of hepatitis $\mathrm{C}$ virus: current knowledge and perspectives. World $\mathrm{J} \mathrm{He}-$ patol 6: 643-651, 2014.

11. Garazzino S, Calitri C, Versace A, et al. Natural history of vertically acquired HCV infection and associated autoimmune phenomena. Eur J Pediatr 173: 1025-1031, 2014.

12. Mizuochi T, Takano $T$, Yanagi $T$, et al. Epidemiologic features of 348 children with hepatitis $\mathrm{C}$ virus infection over a 30 -year period: a nationwide survey in Japan. J Gastroenterol 53: 419-426, 2018.

13. Toyoda H, Kumada T, Takaguchi K, et al. Changes in hepatitis $\mathrm{C}$ virus genotype distribution in Japan. Epidemiol Infect 142: $2624-$ 2628, 2014

14. Suzuki M, Tajiri H, Tanaka Y, et al. Peginterferon therapy in children with chronic hepatitis $\mathrm{C}$ : a nationwide, multicenter study in Japan, 2004-2013. J Pediatr Gastroenterol Nutr 63: 88-93, 2016.

15. Ohmer S, Honegger J. New prospects for the treatment and prevention of hepatitis $\mathrm{C}$ in children. Curr Opin Pediatr 28: 93-100, 2016.

16. Balistreri WF, Murray KF, Rosenthal $P$, et al. The safety and effectiveness of ledipasvir-sofosbuvir in adolescents 12-17 years old with hepatitis $\mathrm{C}$ virus genotype 1 infection. Hepatology 66: 371378, 2017.

17. Wirth S, Rosenthal P, Gonzalez-Peralta RP, et al. Sofosbuvir and ribavirin in adolescents 12-17 years old with hepatitis $\mathrm{C}$ virus genotype 2 or 3 infection. Hepatology 66: 1102-1110, 2017.

18. American Association for the Study of Liver Diseases (AASLD), Infectious Diseases Society of America (IDSA). HCV in Children [Internet]. [cited 2018 Jul 2]. Available from: https://www.HCVGu idance.org

19. Lagging M, Wejstål R, Duberg AS, et al. Treatment of hepatitis $C$ virus infection for adults and children: updated Swedish consensus guidelines 2017. Infect Dis (Lond) 50: 569-583, 2018.

20. Westbrook RH, Dusheiko G. Natural history of hepatitis C. J Hepatol 61: S58-S68, 2014.

21. Honda K, Seike M, Oribe J, et al. Usefulness of semiquantitative PCR-Invader assay for selecting candidates for daclatasvir plus asunaprevir combination therapy among patients with hepatitis C virus genotype 1b. Hepatol Res 48: 255-263, 2018.

22. Jonas MM, Squires RH, Rhee SM, et al. Pharmacokinetics, safety, and efficacy of glecaprevir/pibrentasvir in adolescents with chronic hepatitis C virus: part 1 of the DORA Study. Hepatology. Forthcoming.

23. Younossi ZM, Stepanova M, Marcellin P, et al. Treatment with ledipasvir and sofosbuvir improves patient-reported outcomes: results from the ION-1, -2 , and -3 clinical trials. Hepatology 61: 1798-1808, 2015.

The Internal Medicine is an Open Access journal distributed under the Creative Commons Attribution-NonCommercial-NoDerivatives 4.0 International License. To view the details of this license, please visit (https://creativecommons.org/licenses/ by-nc-nd/4.0/). 